\title{
Adaptive Gas Source Localization Strategies and Gas Distribution Mapping using a Gas-sensitive Micro-Drone
}

\author{
Dipl.-Inf. Patrick Neumann ${ }^{1}$, M.Sc. Inf. Victor Hernandez Bennetts ${ }^{2}$, and Dr.-Ing. Matthias Bartholmai ${ }^{1}$ \\ 'BAM Federal Institute for Materials Research and Testing, \\ Division 8.1 Sensors, Measurement and Testing Methods \\ Unter den Eichen 87, 12205 Berlin \\ E-Mail: patrick.neumann@bam.de \\ E-Mail: matthias.bartholmai@bam.de \\ Phone: (+49/0) 30 - 81043629 / 1912 \\ Fax: (+49/0) 30 - 81041917 \\ ${ }^{2}$ Center for Applied Autonomous Sensor Systems \\ School of Science and Technology, Örebro University, \\ Örebro, SE - 70182 \\ E-Mail: victor.hernandez@oru.se \\ Phone: (+46/0) 19 - 301355 \\ Fax: $(+46 / 0) 19-303463$
}

\begin{abstract}
In this paper we exemplify how to address environmental monitoring tasks with a gas-sensitive micro-drone and present two different approaches to locate gas emission sources. First, we sent the micro-drone in real-world experiments along predefined sweeping trajectories to model the gas distribution. The identification of the gas source location is made afterwards based on the created model. Second, we adapted two bio-inspired plume tracking algorithms that have been implemented so far on ground-based mobile robots. We developed a third bio-inspired algorithm, which is called "pseudo gradient-based algorithm", and compared its perfomance in real-world experiments with the other two algorithms.
\end{abstract}

Keywords: Anemotaxis, chemotaxis, micro UAV, bio-inspired, chemical sensing, gas distribution modeling, gas source localization, gas sensors, mobile sensing system, odor localization, olfaction, plume tracking, quadrocopter.

\section{Introduction}

At present, the release of hazardous and greenhouse gases is an acute threat and mainly responsible for extensive problems, both global (ozone layer depletion and global warming) and local (pollution, poisoning hazards caused by accidents). Gases can be released in many different ways for various reasons: as exhaust gases from traffic or industry, as flue gases from fires, or as a consequence of incidents with chemicals. A fundamental requirement for emission control of, e.g., geodynamically active regions, waste disposals, landfills, capture and storage areas (CCS), industrial sites, and contaminated areas is the availability of measurements of relevant gas concentrations in the area of interest. In order to obtain a truthful representation of the gas distribution, it is essential to collect spatially distributed concentration and wind measurements. Many of these applications are time-critical, i.e., gas leaks should be found as fast as possible. However, due to economical and logistic reasons, a stationary sensor network is in many cases not a viable solution. Accordingly, a quickly deployable, mobile measurement device is needed. The Federal Institute for Materials Research and Testing (BAM), in cooperation with Airrobot $\mathrm{GmbH}$, has developed a measurement system based on a quadrocopter (in the following referred to micro-drone) as part of an R\&D project funded by the Federal Ministry of Economics and Technology (BMWi) [4], [5], [6].

In this paper, we exemplify how to address these environmental monitoring tasks with a gas-sensitive micro-drone (Sec. II) and present two different approaches to locate gas emission sources in an uncontrolled outdoor environment. In the first approach, a gas distribution model is built based on the measurements taken by the micro-drone while following predefined trajectories (Sec. III). The location of the gas source is inferred from this model. In the later approach, we adapt two bio-inspired plume tracking algorithms and present a novel bio-inspired algorithm, which we call "pseudo gradient-based algorithm" (Sec. IV). All three algorithms are based on two principles: chemotaxis and anemotaxis [7]. Chemotaxis refers to a mechanism in which the movement of an organism (or mobile robot) is determined by the distribution of chemical compounds, most often by the concentration gradient of one or more volatiles. Anemotaxis instead refers to a mechanism in which the movement of an organism (or mobile robot) is determined by the perceived airflow. Ultimately, we compare the perfomance of the three plume tracking algorithms in real-world experiments with each other and draw conclusions (Sec. V). Kowadlo and Russell give in [1] an excellent summary of the existing algorithms. 
However, all plume tracking algorithms have been implemented and tested so far on ground-based mobile robots under simplified environmental conditions. Thus, a main contribution of this work is the development of gas source localization strategies for a gas-sensitive micro-drone and the validation in several real-world experiments.

\section{Gas-sensitive Micro-Drone}

The Airrobot AR100-B micro-drone (Fig. 1) has a diameter of $1 \mathrm{~m}$ and is operated by four brushless electric motors. The micro-drone can precisely navigate to a certain region of interest for remote sensing without endangering persons in critical areas. The maximum payload mass amounts to $200 \mathrm{~g}$ with a total flight mass of about $1.3 \mathrm{~kg}$. The micro-drone was modified to incorporate gas-sensitive devices as payloads. An e-nose capable of accommodate four commercially available metal oxide (MOX) sensors and an electrochemical cell is used as a gas-sensitive payload (i.a., Figaro TGS 2611 - methane $\left[\mathrm{CH}_{4}\right]$ ). The maximum flight time is about $20-30 \mathrm{~min}$. The micro-drone can withstand a maximum wind speed of $8 \mathrm{~m} / \mathrm{s}$. The flight control relies on an on-board inertial measurement unit (IMU). It consists of a three axis accelerometer and a three axis rotation rate sensor. Magnetic field sensor (compass) and GPS improve the accuracy of the IMU, and are used to compensate for the sensor drift. A barometric pressure sensor is used to control the altitude of the micro-drone. Communication with the ground station is established by a wireless radio link. Data packets can include control instructions or data coming from the micro-drone's on-board sensors. The operating distance of the remote control and communication link is $1 \mathrm{~km}$. Due to the restrictions imposed by the platform, the micro-drone doesn't carry any wind sensing modalities. Instead, wind measurements are estimated by fusing the different on-board sensors to compute the parameters of the wind triangle [6]. The wind triangle is commonly used in navigation and describes the relationships between the flight vector $v$, the ground vector $w$, and the wind vector $u$. The micro-drone can be operated manually or in GPS mode, e.g., by autonomous waypoint following.

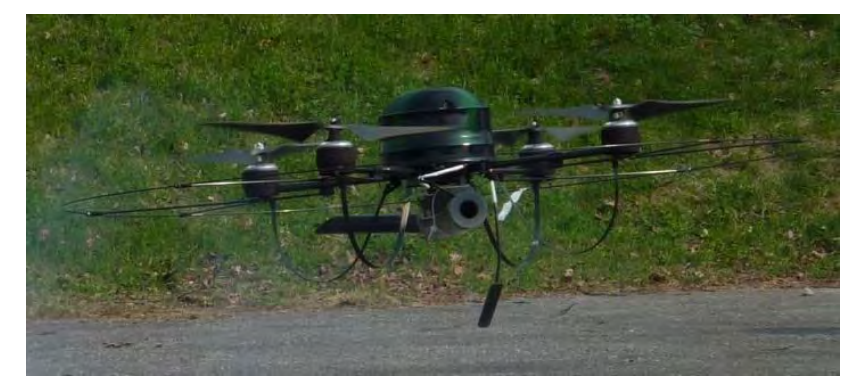

Fig. 1. The Airrobot AR100-B micro-drone equipped with the e-nose.

\section{Gas Distribution Modeling with Predefined Trajectory}

Gas distribution mapping can be used in a number of relevant application areas where a better understanding of the gas dispersion is needed, such as environmental monitoring and safety and security related fields [8]. To estimate the gas distribution model, we use the Kernel DM+V/W algorithm introduced by Reggente and Lilienthal [9], which is an extension of Kernel DM+V [10] that also considers wind information to compute the gas distribution model. The gas distribution model provides the basis for data interpretation and evaluation of results of autonomous real-world outdoor experiments performed on the BAM Test Site 'Technical Safety' (BAM TTS).

The input to this algorithm is a set $D=\left\{\left(x_{1}, r_{1}, v_{1}\right), \ldots,\left(x_{n}, r_{n}, v_{n}\right)\right\}$ of gas sensor measurements $r_{i}$ and airflow measurements $v_{i}$ collected at locations $x_{i}$. The output is a grid model that computes an estimate of a confidence, as well as the distribution mean and variance for each cell. We use the 2D version of the Kernel $\mathrm{DM}+\mathrm{V} / \mathrm{W}$ algorithm to avoid the higher computational complexity of the $3 \mathrm{D}$ Kernel DM+V/W algorithm [11] and because the limited battery capacity of the micro-drone does not permit a full 3D search. Moreover, in the experiments, the micro-drone was kept at an approx. constant height in a single 2D plane.

\section{A. Real-world Experiments}

Experimental Setup - The experiments were carried out in a natural environment on the BAM TTS over an area of $5 \times 5 \mathrm{~m}^{2}$ (Fig. 2). The area is surrounded by trees and testing buildings, which introduced a 
certain level of turbulence. The sensors of the e-nose were sampled at a frequency of $8 \mathrm{~Hz}$. The step size of the micro-drone in $x$ and $y$ direction was set to $1.0 \mathrm{~m}$. The flight speed of the micro-drone between the measurement positions was set to $1 \mathrm{~m} / \mathrm{s}$. The micro-drone was programmed to explore the experimental areas following a sweeping trajectory using autonomous control software. In Fig. 2, the starting point is illustrated by the red star. Because of the low flight height of about $1.5-2.0 \mathrm{~m}$, the height of the microdrone was corrected manually during the experiments. $\mathrm{A} \mathrm{CH}_{4}$ (99.5\%-pure methane) gas cylinder connected to a pressure reducing valve was used as the gas source. The outlet of the gas source was extended with a small tube, which was attached to a fan in order to spread the analyte away from the cylinder. The air current introduced by the fan also prevented the $\mathrm{CH}_{4}$ to immediately rise up to the atmosphere when released. The micro-drone was set to autonomous waypoint mode directly after takeoff, which started the experiment.

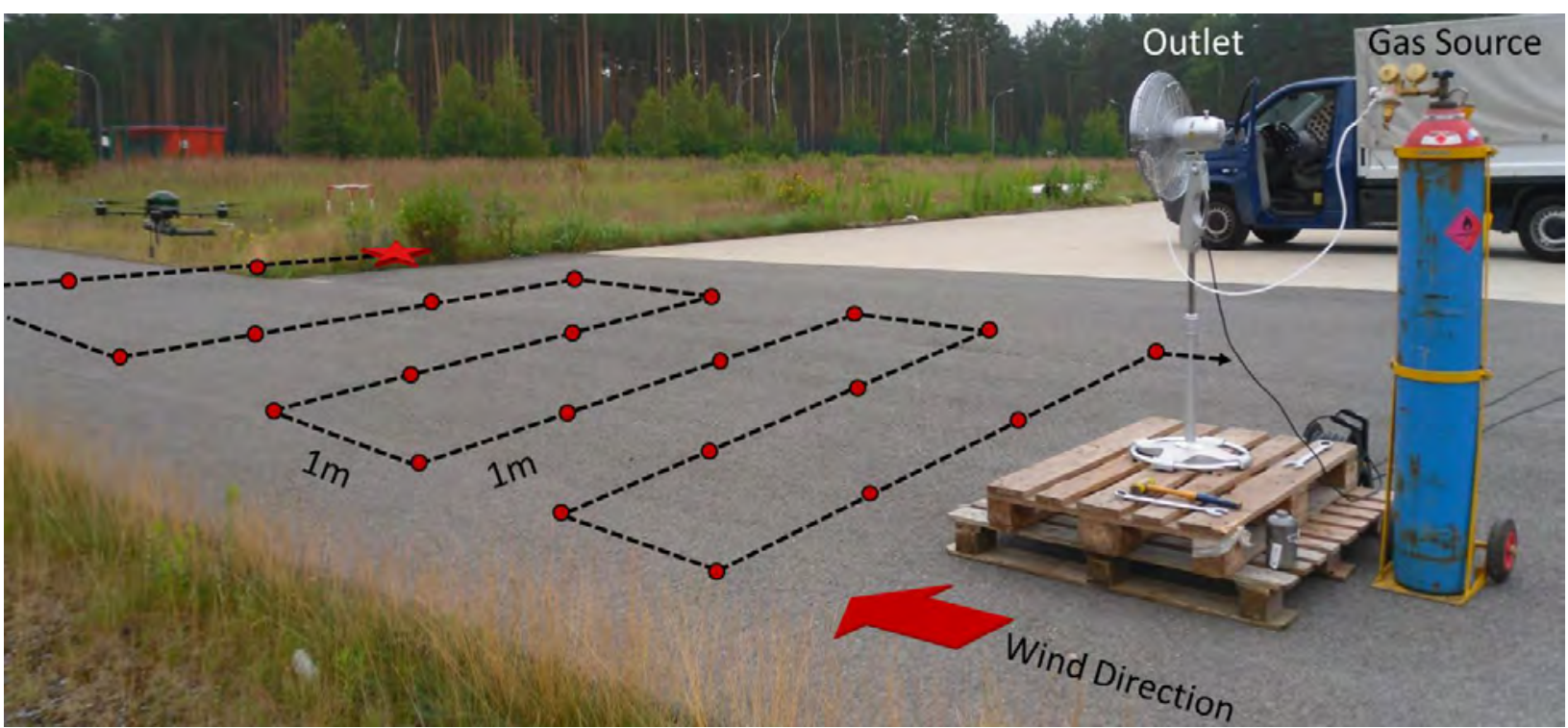

Fig. 2. Experimental environment and setup of the experiments performed on the BAM TTS. The pre-programmed flight trajectory of the micro-drone is shown with a dashed line. The red dots indicate the sampling locations on the trajectory, whereas the starting point is indicated by the red star. The red arrow illustrates the main wind direction during the experiments. The combination of a $\mathrm{CH}_{4}$ gas bottle and a fan was used as the emission source.

Results - The maps were created using the Kernel DM+V/W algorithm with $\gamma$ heuristically set to $0.2 \mathrm{~s}$, a cell size $c$ of $0.25 \mathrm{~m}$, and a kernel width $\sigma$ of $1.0 \mathrm{~m}$. Fig. 3 shows the results of the experiments. The wind conditions measured with the micro-drone were very stable during all three trials (Tab. 1): the average wind speed was in the range of approx. $1.4-1.7 \mathrm{~m} / \mathrm{s}$ and the average wind direction was between 246 and $259^{\circ}$. The degree of stability for each run is given by the circular variance [12] which lay around 0.21 and 0.32 . Additionally, the airflow maps in Fig. 3 reflect the stability of the local wind conditions.

The gas emission of the artificial gas source used in the experiments was started in the beginning of each trial. The artificial gas source had a more or less constant release rate over the period of the experiment as the pressure reducing valve was set up at a constant pressure of approx. 5 bar. The artificial gas source and the stable wind conditions on the BAM TTS with the relative high wind speeds permitted to form instantly a reproducible plume moving away from its source (Fig. 3).

\begin{tabular}{c|c|c|c|c|c}
\hline \hline Trial & \#Samples & $\begin{array}{c}\text { Mean Wind } \\
\text { Speed }(\mathrm{m} / \mathrm{s})\end{array}$ & $\begin{array}{c}\sigma \\
(\mathrm{m} / \mathrm{s})\end{array}$ & $\begin{array}{c}\text { Mean Wind } \\
\text { Direction }\left(^{\circ}\right)\end{array}$ & $\begin{array}{c}\text { Circular } \\
\text { Variance }\end{array}$ \\
\hline 1 & 9,938 & 1.38 & \pm 0.71 & 247 & 0.32 \\
2 & 7,871 & 1.65 & \pm 0.82 & 246 & 0.21 \\
3 & 10,939 & 1.57 & \pm 0.82 & 259 & 0.26 \\
\hline \hline
\end{tabular}

Tab. 1. Wind conditions on the BAM TTS.

Reproducibility is given for trial $1-3$ as the qualitative and quantitative results remain more or less constant in all created maps. Small variations in the plume propagation can be explained by changes in the wind field during each experimental run. It can be seen, that the produced gas distribution maps match excellent to their corresponding airflow maps. The results from the last trial differ more 
pronouncedly as the airflow map shows stronger variations in the wind field. The areas of high predictive mean and high predictive variance in trial \#2 and \#3 accurately indicate the location of the gas source (at position $(0,3) \mathrm{m}$ ), which can be seen in Figs. $\mathbf{3}(\mathbf{b})$ and $\mathbf{3}(\mathbf{c})$. As the micro-drone had to pass the gas source very close in the last sweep, it happened that the height of the micro-drone had to be changed manually to avoid a collision with the fan. This occurred in trial \#1 due to the inaccuracies given by the GPS receiver. Therefore, the last sweep in trial \#1 was performed in a height of approx. $1 \mathrm{~m}$ directly above the fan and the plume, respectively. This resulted in low concentrations measurements close to the source, which displaced the high concentration areas approx. $2-4 \mathrm{~m}$ further downwind.
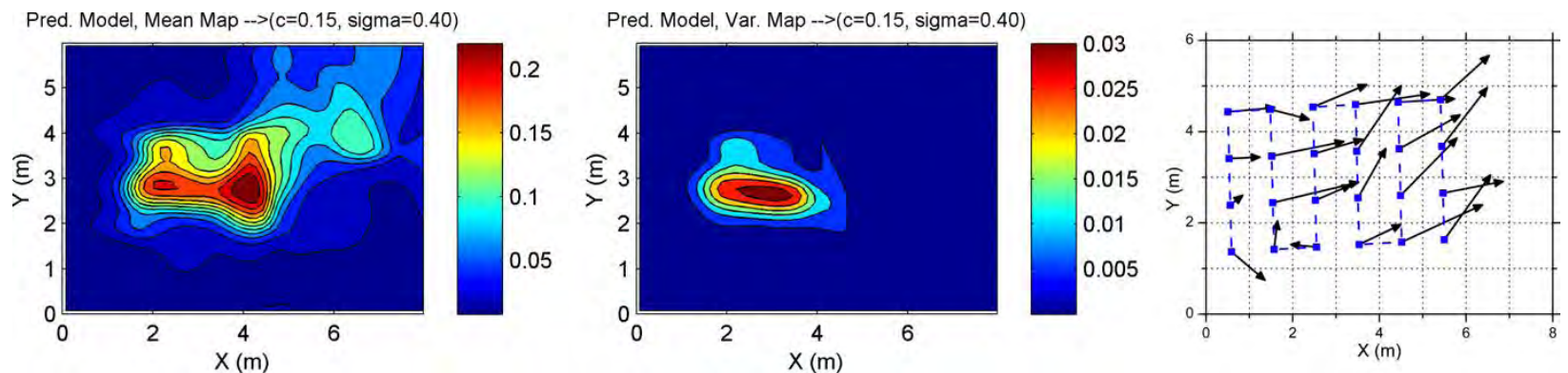

(a) Trial \#1; $\mathrm{CH}_{4}$
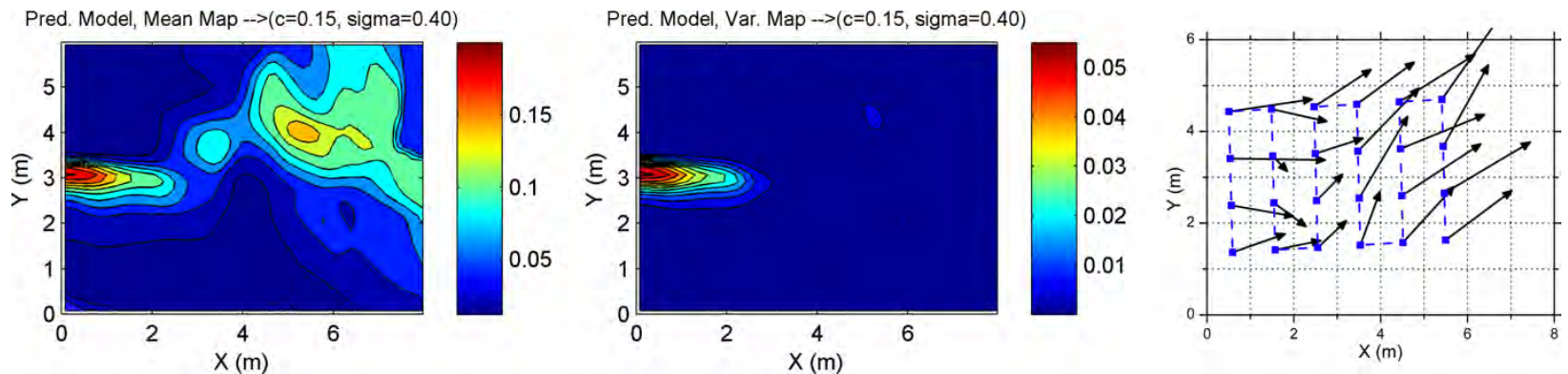

(b) Trial \#2; $\mathrm{CH}_{4}$
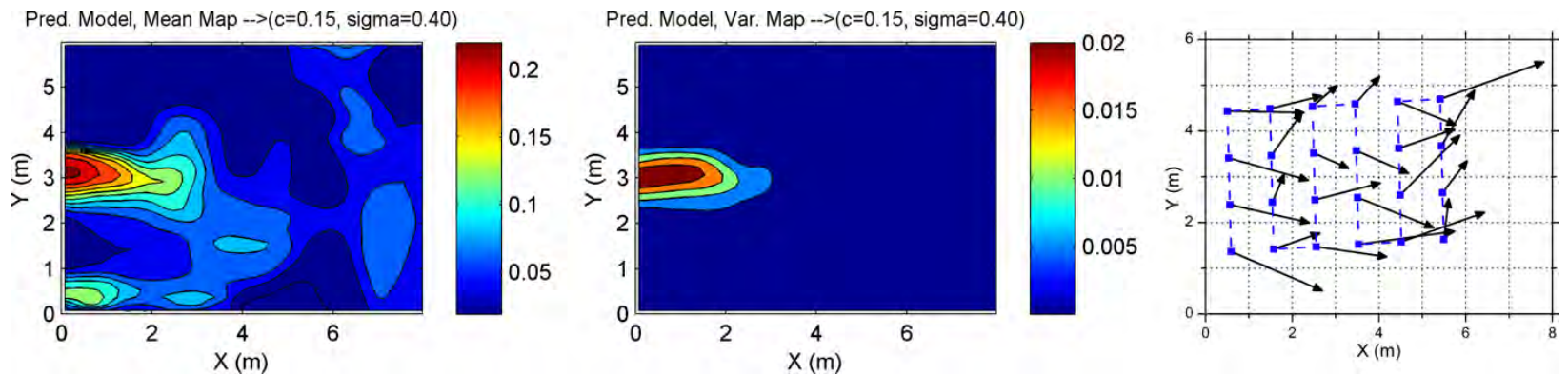

(c) Trial \#3; $\mathrm{CH}_{4}$

Fig. 3. Predictive mean (left) and variance map (middle) of the gas distribution of the (a) 1st, (b) 2nd, and (c) 3rd trial. The right figures show the corresponding mean airflow maps. The gas source was located at approx. at position $(0,3) \mathrm{m}$. The starting position of the micro-drone is located at position approx. $(5.5,1.5) \mathrm{m}$. The concentration value of $\mathrm{CH}_{4}$ is given in $\%$ by volume.

\section{Plume Tracking using a Gas-sensitive Micro-Drone}

Three of the most promising plume tracking algorithms were implemented and adapted accordingly to meet the system characteristics of the gas-sensitive micro-drone (e.g., the minimum step size limitation of $1 \mathrm{~m}$ ): the surge-cast algorithm (a variant of the silkworm moth algorithm) [14], the zigzag/dung beetle algorithm [15], and a newly developed algorithm called "pseudo gradient-based algorithm". They were successfully tested in real-world experiments. However, no in depth validations of these algorithms were performed as different researchers have done this extensively before in simulation and wind tunnel experiments, for example Lochmatter [14] and Russell et al. [16], to name but a few.

Reactive plume tracking algorithms can be separated into three phases [1]: (1) plume acquisition (find the plume), (2) plume tracking (move the robot reactively along/within the plume), and (3) source declaration 
(verify the identified source). However, the source declaration phase was not considered within this work. The plume acquisition phase can be done using a, e.g., randomized or systematic search. Here, the following systematic search algorithm, called sweeping, was implemented:

- collect gas sensor and wind measurements while keeping the micro-drone at a fixed position for a prolonged time (here: $20 \mathrm{~s}$ );

- average the gas concentration and wind measurements over the measurement time;

- if the averaged gas concentration $\bar{c}$ is below a given threshold $t h_{c}\left(\bar{c} \leq t h_{c}\right)$, make a step orthogonal to the wind direction: if this new position will be outside the search space, make one step in upwind direction and change the sweeping direction;

- if the averaged gas concentration $\bar{c}$ is above a given threshold $t h_{c}\left(\bar{c}>t h_{c}\right)$, change to the plume tracking phase; otherwise continue with the first step.

Note that the calculation of next measuring positions is done using great circle navigation formulae [13], which need the current position of the micro-drone, an azimuth direction angle, and a defined step size.

\section{A. Surge-Cast Algorithm}

Lochmatter and Martinoli presented in [14] the surge-cast algorithm. It is a combination of plume tracking strategies used by the silkworm moth. The surge-cast algorithm for the gas-sensitive micro-drone works as follows:

- collect gas sensor and wind measurements while keeping the micro-drone at a fixed position for a prolonged time (here: $20 \mathrm{~s}$ );

- average the gas concentration and wind measurements over the measurement time;

- if the averaged gas concentration $\bar{c}$ is above the threshold $t h_{c}\left(\bar{c}>t h_{c}\right)$, perform an upwind surge (move the micro-drone one step straight forward in wind direction);

- if the averaged gas concentration $\bar{c}$ is below the threshold $t h_{c}\left(\bar{c} \leq t h_{c}\right)$, carry out casting in crosswind direction with increasing step size: \# no detection events $\times$ step size (return to sweeping, if no concentration was detected after a defined number of steps); and repeat with the first step.

\section{B. Zigzag/Dung Beetle Algorithm}

The following zigzagging algorithm was developed in analogy to the one presented in [15]. The algorithm changes its behavior from sweeping into zigzagging once the averaged gas concentration $\bar{c}$ is above the threshold $t h_{c}$.

- collect gas sensor and wind measurements while keeping the micro-drone at a fixed position for a prolonged time (here: $20 \mathrm{~s}$ );

- average the gas concentration and wind measurements over the measurement time;

- if the averaged gas concentration $\bar{c}$ is above the threshold $t h_{c}\left(\bar{c}>t h_{c}\right)$ or the new position after the movement of the micro-drone will be outside the search space, set $\alpha$ to $-\alpha$;

- if the averaged gas concentration $\bar{c}$ is below the threshold $t h_{c}\left(\bar{c} \leq t h_{c}\right)$ for a predefined number of steps, return to sweeping;

- turn the micro-drone $\alpha^{\circ}$ from upwind direction and move a step forward; and repeat with the first step. 


\section{Pseudo Gradient-based Algorithm}

As the rotors of the micro-drone introduce strong turbulence, measuring a local concentration gradient with spatially separated sensors is not feasible. Instead a new measuring strategy was developed, which basically splits up one measuring position into two spatially separated ones. The step size in upwind direction $d_{u p}$ was set to $d_{\text {cross }}+d_{\text {cross }} / 2$ in order to respect the minimum step size of the micro-drone of 1 $\mathrm{m}$ and to progress faster to the source. After the gas sensor has detected a concentration above a threshold $t h_{c}$, the following procedure is repeated (requires $\alpha=90^{\circ}$ ):

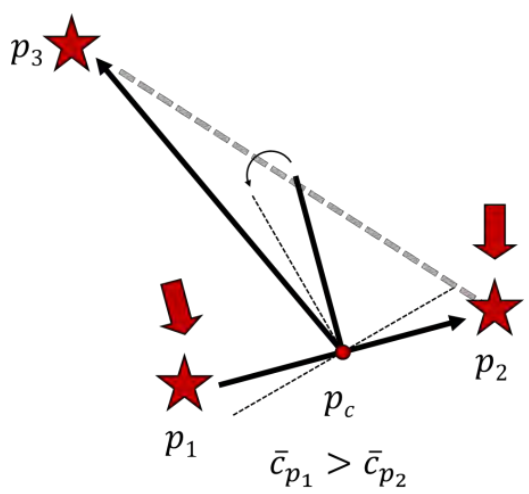

(a)

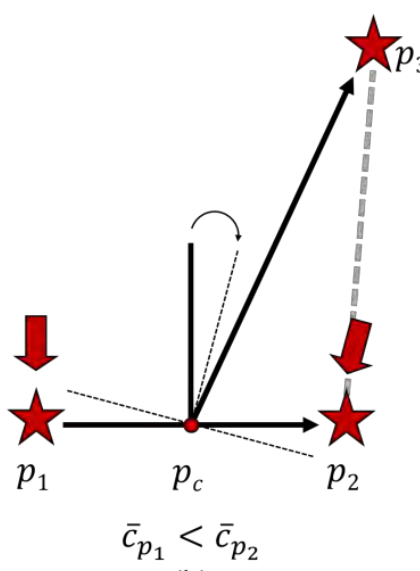

(b)

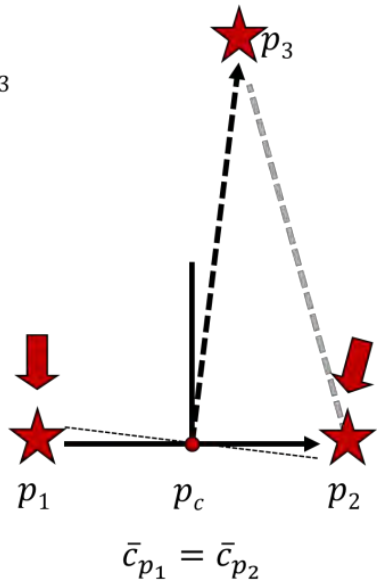

(c)

Fig. 4. The three different cases of the gradient-based algorithm: (a) $\bar{c}_{p 1}>\bar{c}_{p 2}$, (b) $\bar{c}_{p 1} \leq \bar{c}_{p 2}$, and (c) $\bar{c}_{p 1}=\bar{c}_{p 2}$. The case $\bar{c}_{p 1}=\bar{c}_{p 2}=$ 0 restarts the plume acquisition phase. The red stars indicate the measuring positions, the red arrows illustrate the averaged wind directions, and the gray dotted line is the shortened flight path of the micro-drone.

- collect gas sensor and wind measurements while keeping the micro-drone at position $p_{1}$ for a prolonged time (here: $20 \mathrm{~s}$ );

- calculate the averaged gas concentration $\bar{c}_{p 1}$ and wind direction $\bar{u}_{d i r ; p 1}$ over the measurement time;

- turn the micro-drone $\alpha^{\circ}$ from upwind direction and move a step forward;

- collect gas sensor and wind measurements while keeping the micro-drone at position $p_{2}$ for a prolonged time;

- calculate the averaged gas concentration $\bar{c}_{p 2}$ and wind direction $\bar{u}_{d i r ; p 2}$ over the measurement time;

- calculate the concentration gradient (an average concentration $\bar{c}$ below the threshold $t h_{c}$ will be set to 0 ; if $\bar{c}_{p 1}=\bar{c}_{p 2}=0, \alpha$ is set to $-\alpha$ and sweeping is restarted):

$$
\operatorname{grad}\left(\bar{c}_{p 1}, \bar{c}_{p 2}\right)=\frac{\bar{c}_{p 1}}{\bar{c}_{p 1}+\bar{c}_{p 2}}, \operatorname{grad}\left(\bar{c}_{p 1}, \bar{c}_{p 2}\right) \in[0,1]
$$

- if $\operatorname{grad}\left(\bar{c}_{p 1}, \bar{c}_{p 2}\right)>0.5$ set $\alpha$ and $\beta$ to $-\alpha$ and $-\beta$ accordingly and calculate the new measuring position $p_{3}$ based on the averaged wind direction determined at position $p_{1}$ and the center position $p_{c}$ using the following equations (Fig. 4(a)):

$$
\delta=\bar{u}_{d i r ; p 1}+\beta \cdot 2 \cdot\left(\operatorname{grad}\left(\bar{c}_{p 1}, \bar{c}_{p 2}\right)-0.5\right)
$$

- if $\operatorname{grad}\left(\bar{c}_{p 1}, \bar{c}_{p 2}\right)<0.5$ calculate the new measuring position $p_{3}$ based on the averaged wind

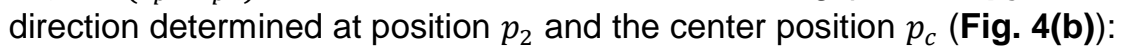




$$
\delta=\bar{u}_{d i r ; p 2}+\beta \cdot 2 \cdot\left(\operatorname{grad}\left(\bar{c}_{p 1}, \bar{c}_{p 2}\right)-0.5\right)
$$

- if $\operatorname{grad}\left(\bar{c}_{p 1}, \bar{c}_{p 2}\right)=0.5$ calculate the new measuring position $p_{3}$ based on the averaged wind direction of both measuring points $p_{1}$ and $p_{2}$ and the center position $p_{c}$ (Fig. 4(c)):

$$
\delta=\bar{u}_{d i r ; p 1, p 2}
$$

- fly the micro-drone autonomously directly from position $p_{2}$ to position $p_{3}$ and repeat with the first step.

\section{Real-world Experiments}

Experimental Setup - All plume tracking experiments were carried out in a $20 \times 16 \mathrm{~m}^{2}$ wide open outdoor area surrounded by trees and testing buildings, which introduced a certain level of turbulence (Fig. 5). The experimental setup was identical with the one described in Sec. III-B with the following exceptions: The step size of the micro-drone was set to $1.5 \mathrm{~m}$ for the surge-cast and the pseudo gradientbased algorithm and $2.0 \mathrm{~m}$ for the zigzag algorithm, respectively. The parameter $\alpha$ of the zigzag algorithm was set to $60^{\circ}$ in the first and $75^{\circ}$ in the second run. The parameter $\beta$ of the pseudo gradient-based algorithm was set to $60^{\circ}$. The gas source was placed approximately at position $(3.2,5.7) \mathrm{m}$ from the bottom left corner. The corresponding algorithm was activated directly after take-off, which started the experiment.

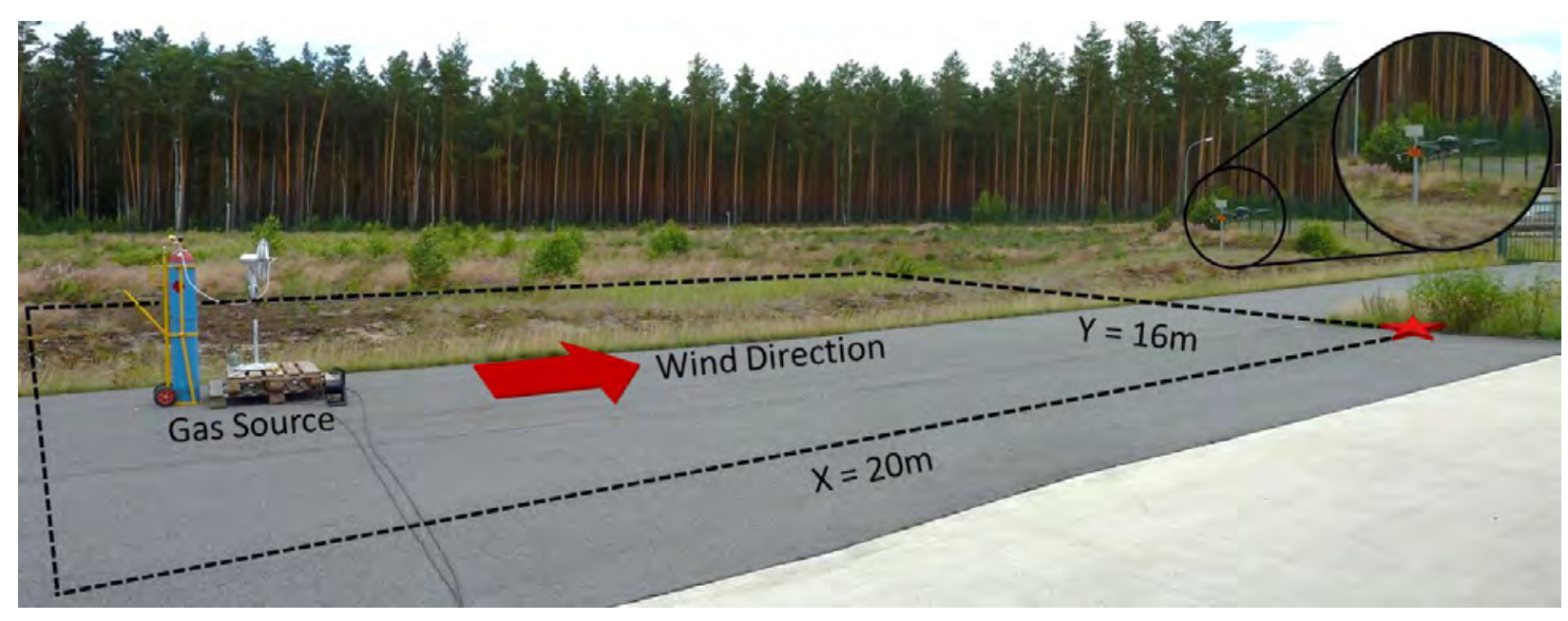

Fig. 5. Setup of the plume tracking experiments: The experimental area was set to $20 \times 16 \mathrm{~m}^{2}$ with the gas source positioned approximately at position $(3.2,5.7) \mathrm{m}$ from the bottom left corner. The red arrow illustrates the main wind direction during the experiments. The start position of the micro-drone is indicated by the red star. The micro-drone can be seen in the image enlargement in the upper right corner of the figure.

Results - The wind conditions during the experiments permitted to perform a total of 7 successful runs: the surge-cast and zigzag algorithms were conducted twice, whereas the gradient-based approach was performed three times. The circular variance is used here to describe the degree of turbulence (stability of the wind) during the different experimental runs [12].

The distance overhead $d_{0}$ is used as the performance metric to compare the plume tracking algorithms experiments with each other [17] and is defined as:

$$
d_{0}=\frac{d_{t}}{d_{u}}
$$

where $d_{t}$ denotes the effectively traveled distance by the micro-drone and $d_{u}$ the upwind distance. The upwind distance $d_{u}$ is calculated using the Euclidean distance between the position, where the microdrone measures for the first time an average concentration above the threshold, and the source position (minus the distance at the end of the experiment). Therefore, it represents the shortest path a robot would take if the source location is known. 


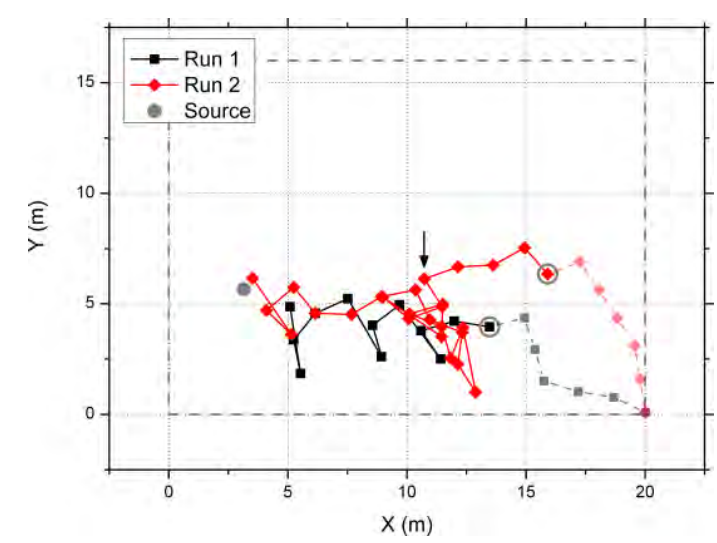

(a)

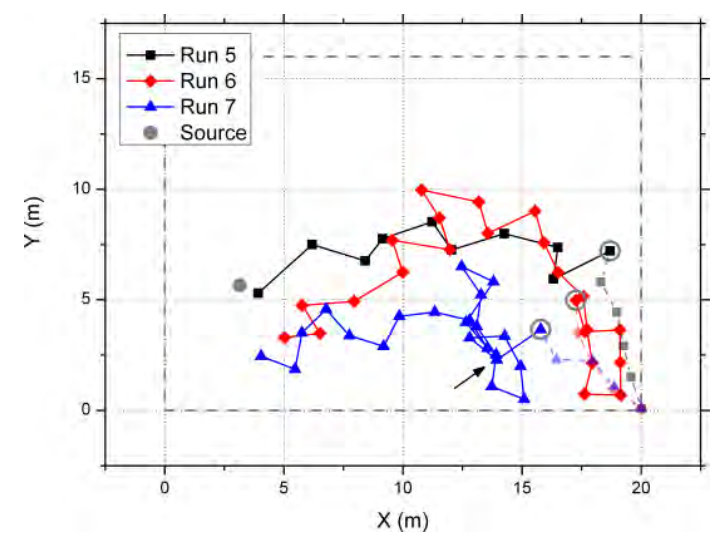

(c)

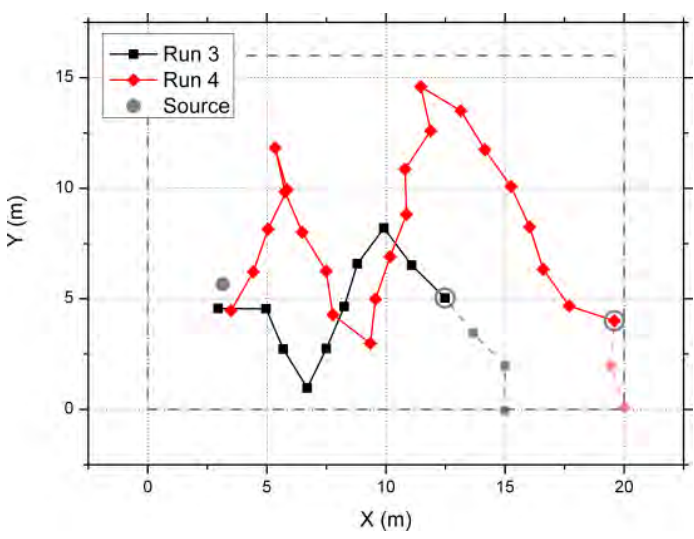

(b)

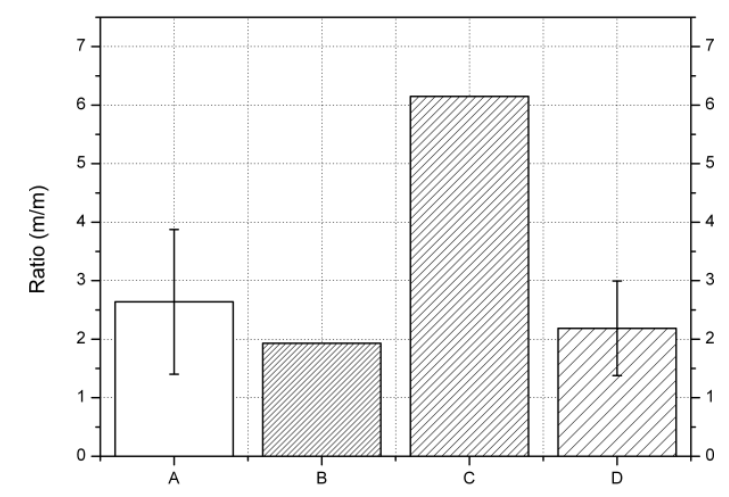

(d)

Fig. 6. Trajectories of the micro-drone including the plume acquisition phase using (a) the surge-cast, (b) the zigzag, and (c) the pseudo gradient-based algorithm. The experimental area is illustrated by the gray dotted line. The source position is indicated by the gray point. The gray circles mark the position of the first gas concentration measurements above the threshold. The position, where the wind direction started turning up to $180^{\circ}$ is indicated with black arrows (in run \#2 and \#8). (d) Comparison of the traveled distance $d_{t} /$ the upwind distance $d_{u}$ (mean with 95\% confidence interval) of the A: surge-cast, B: the zigzag $\left(\alpha=60^{\circ}\right)$, C: the zigzag $\left(\alpha=75^{\circ}\right)$, and $\mathrm{D}$ : the pseudo gradient-based algorithm (the traveled distance needed to acquire the plume was not considered here).

Figs. 6(a) - 6(c) show the trajectories produced by the micro-drone. However, the plume acquisition phase was excluded from the evaluation to make the results rudimentary comparable to each other. Particularly noticeable are the runs \#2 (surge-cast) and \#7 (gradient-based). Here, the wind direction varied significantly during the runs precluding the micro-drone to make further progress for some time. This is indicated in Fig. 6 with black arrows. Tab. 2 shows a compilation of the most relevant experimental results.

Reproducible conditions in a wind tunnel using a laminar airflow permitted to generate approx. the same plume structure (width, intermittency, and concentrations) within the experimental setup. This cannot be achieved in the real-world. Especially since the wind direction is changing permanently about a few degrees. Even the experimental setup introduced additional turbulence. Another problem is the different chosen $\alpha$ parameters used with the zigzag algorithm. The overlapping confidence intervals, the small number of experiments, and the permanent changing structure of the plume do not permit to make a complete statistical evaluation of the algorithms.

\begin{tabular}{c|cc|cc|ccc}
\hline \hline & \multicolumn{2}{|c|}{ Surge-Cast } & \multicolumn{2}{|c|}{ Zigzag } & \multicolumn{3}{c}{ Gradient-based } \\
\hline Run & 1 & 2 & 3 & 4 & 5 & 6 & 7 \\
Traveled Distance $d_{t}(\mathrm{~m})$ & 21.01 & 41.78 & 18.06 & 44.18 & 23.97 & 37.05 & 34.58 \\
Distance Overhead $(\mathrm{m} / \mathrm{m})$ & 2.01 & 3.27 & 1.93 & 6.15 & 1.53 & 2.62 & 2.70 \\
Mean & \multicolumn{2}{|c|}{2.64} & \multicolumn{2}{|c|}{4.04} & \multicolumn{3}{c}{2.19} \\
95\% Confidence Interval & \multicolumn{2}{|c|}{$(1.40,3.88)$} & \multicolumn{2}{|c|}{$(1.12,6.96)$} & \multicolumn{3}{|c}{$(1.38,3.00)$} \\
\hline \hline
\end{tabular}

Tab. 2. Results of the plume tracking experiments (excluding the plume acquisition phase). 
However, the results demonstrate that plume tracking can be performed in a real-world outdoor environment with a reasonable degree of turbulence. The micro-drone was able to reacquire the plume even during varying wind conditions. Our results indicate that the surge cast algorithm works better than pure casting (zigzagging; $\alpha=75^{\circ}$ ). Further, it can be seen that the pseudo gradient-based algorithm is at least as efficient in the real-world as the surge-cast algorithm.

\section{Summary and Conclusion}

"Probably one of the most crucial aspects of research in mobile robot olfaction is the design of the experiments that enable the study and development of airborne chemical monitoring systems. A major technical difficulty is that the dispersion of chemicals in natural environments is difficult to observe since most chemicals produce an invisible plume. In a non-artificial environment, the plume evolution is also difficult to predict a priori due to the chaotic dispersal of gas. A second major difficulty is that environmental conditions are often very variable and therefore experiments are hard to repeat. Thus, it is difficult to obtain ground truth that can be used to validate experimental results. In order to overcome this limitation, experiments are often carried out under controlled conditions that limit variations from the expected ground truth plume behavior and thus increase repeatability of the experiments. On the other hand, it is hard to predict how the results obtained in such experiments extend to uncontrolled environments. It is very important in the mobile robot olfaction domain that great care is used in describing the experimental setup in which the claimed results are obtained, and it should be avoided to state that results obtained in an environment with, e.g., steady and controlled airflow trivially extend to more general and complex environments." [7]

Gas distribution modeling by an autonomous flying gas-sensitive micro-drone in an uncontrolled environment is an extremely challenging field of research. Even though, the explored areas presented within this chapter are not of considerable size, the application of the Kernel DM+V/W algorithm in combination with the estimation of the wind vector using a micro-drone is very promising with respect to the results of the presented real-world gas distribution mapping experiments. The experiments showed that qualitative and quantitative reproducibility is given, when the wind and weather conditions remain constant over a longer time period. However, we showed that the gas-sensitive micro-drone can be used in environmental monitoring scenarios to detect gas leaks.

Using sweeping trajectories to build detailed gas distribution maps over large areas is time consuming. The batteries of the micro-drone only provide power for approx. 20 min (equipped with payload). New battery technologies may overcome this limitation in the near future. However, adaptive sampling strategies have to be developed or a swarm of multiple gas-sensitive micro-drones has to be deployed. The latter aspect has to be taken with a pinch of salt: A single gas-sensitive micro-drone already introduces plenty of turbulences in the exploration area and a swarm may destroy important evidence.

The results of the plume tracking experiments demonstrate that plume tracking with a gas-sensitive micro-drone can be done in the real-world when particular environmental conditions are available. The micro-drone was able to reacquire the plume even during varying wind conditions. Our initial results indicate that the pseudo gradient-based algorithm is at least as efficient in the real-world as the surgecast algorithm. Simulation experiments will follow to determine the performance of the pseudo gradientbased algorithm in comparison to the other two bio-inspired algorithms.

\section{Acknowledgment}

The authors thank the participating colleagues from BAM, Freie Universität Berlin, and Örebro University. The authors also would like to express their gratitude to BMWi (MNPQ Program; file number 28/07) and Robotdalen (Gasbot; project number 8140) for funding the research.

\section{References}

[1] G. Kowadlo and R. A. Russell. Robot Odor Localization: A Taxonomy and Survey. Int. J. Rob. Res., 27(8):869-894, 2008.

[2] M. Vergassola, E. Villermaux, and B. Shraiman. 'Infotaxis' as a strategy for searching without gradients. In: Nature 445 (2007), January, Nr. 7126, pp. 406-409

[3] M. Vergassola, E. Villermaux, and B. Shraiman. Supplementary Materials for "Infotaxis: searching without gradients". In: Nature 445 (2007), January, Nr. 7126 
[4] P. Neumann, M. Bartholmai, J. H. Schiller, M. Manolov, and B. Wiggerich, Self-Optimizing Search and Characterization of Gaseous Hazardous Substance Sources using a Micro-Drone: A new Approach to determine Wind Speed and Direction, in: IEEE International Workshop on Robotic and Sensors Environments (ROSE 2010), Phoenix, AZ, USA, 2010.

[5] M. Bartholmai and P. Neumann, Adaptive ortsaufgelöste Gaskonzentrationsmessung mit einer Mikrodrohne, tm Technisches Messen, Oldenbourg Wissenschaftsverlag GmbH, 2011, 78, 470-478.

[6] P. Neumann, S. Asadi, J. H. Schiller, A. J. Lilienthal, and M. Bartholmai, Micro-Drone for Wind Vector Estimation and Gas Distribution Mapping, IEEE Robotics and Automation Magazine, to appear 2012.

[7] V. Hernandez Bennetts, A. J. Lilienthal, P. Neumann, and Marco Trincavelli, Mobile Robots for Localizing Gas Emission Sources on Landfill Sites: Is Bio-Inspiration the Way to Go?, Frontiers in Neuroengineering, 2011.

[8] A. Loutfi, S. Coradeschi, A. J. Lilienthal, and J. Gonzalez. Gas distribution mapping of multiple odour sources using a mobile robot. Robotica, 27(2):311-319, 2008.

[9] M. Reggente and A. J. Lilienthal, "Using Local Wind Information for Gas Distribution Mapping in Outdoor Environments with a Mobile Robot," in Proceedings of IEEE Sensors, 2009, pp. 1715-1720.

[10] A. J. Lilienthal, M. Reggente, M. Trincavelli, J. L. Blanco, and J. Gonzalez. A Statistical Approach to Gas Distribution Modelling with Mobile Robots - The Kernel DM+V Algorithm. In Proceedings of the IEEE/RSJ International Conference on Intelligent Robots and Systems (IROS), pages 570-576, October 11 - October 152009.

[11] M. Reggente and A. J. Lilienthal. The 3D-Kernel DM+V/W Algorithm: Using Wind Information in Three Dimensional Gas Distribution Modelling with a Mobile Robot. In Proceedings of IEEE Sensors, 2010.

[12] G. L. Gaile and J. E. Burt. Directional Statistics. Concept s and Techniques in Modern Geography, volume 25 of Geo Books. Institute of British Geographers, Norwich, England, 1980.

[13] E. Williams. Aviation Formulary V1.46. http://williams.best.vwh.net/, 04/2011.

[14] T. Lochmatter and A. Martinoli. Tracking Odor Plumes in a Laminar Wind Field with Bio-Inspired Algorithms. In 11th International Symposium on Experimental Robotics 2008 (ISER 2008), volume 54, pages 473-482. Springer, 2009.

[15] H. Ishida, K. Suetsugu, T. Nakamoto, and T. Moriizumi. Study of autonomous mobile sensing system for localization of odor source using gas sensors and anemometric sensors. Sensors and Actuators A, 45:153-157, 1994.

[16] R. Russell, A. Bab-Hadiashar, R. Shepherd, and G. Wallace. A comparison of reactive chemotaxis algorithms. In Robotics and Autonomous Systems, volume 45, pages 83-97, 2003.

[17] T. Lochmatter. Bio-Inspired and Probabilistic Algorithms for Distributed OdorSource Localization using Mobile Robots. PhD thesis, EPFL, Lausanne, Switzerland, February 2010.

[18] A. J. Lilienthal, T. Duckett, F. Werner, and H. Ishida. Indicators of Gas Source Proximity using Metal Oxide Sensors in a Turbulent Environment. In Proceedings of the IEEE / RAS-EMBS International Conference on Biomedical Robotics and Biomechatronics (Biorob), February 20-22 2006. 